\title{
Association between objectively assessed sedentary time and physical activity with metabolic risk factors among people with recently diagnosed type 2 diabetes
}

\author{
Andrew J. M. Cooper • Soren Brage • Ulf Ekelund • \\ Nicholas J. Wareham • Simon J. Griffin • \\ Rebecca K. Simmons
}

Received: 23 April 2013 / Accepted: 13 September 2013 / Published online: 3 October 2013

(C) The Author(s) 2013. This article is published with open access at Springerlink.com

\begin{abstract}
Aims/hypothesis The aim of our study was to examine the associations between sedentary time (SED-time), time spent in moderate-to-vigorous-intensity physical activity (MVPA), total physical activity energy expenditure (PAEE) and cardiorespiratory fitness with metabolic risk among individuals with recently diagnosed type 2 diabetes.

Methods Individuals participating in the Anglo-Danish-Dutch Study of Intensive Treatment in People with Screen Detected Diabetes in Primary Care (ADDITION)-Plus trial underwent measurement of SED-time, MVPA and PAEE using a combined activity and movement sensor $(n=394)$, and evaluation of cardiorespiratory fitness $(n=291)$ and anthropometric and metabolic status. Clustered metabolic risk was calculated by summing standardised values for waist circumference, triacylglycerol, $\mathrm{HbA}_{1 \mathrm{c}}$, systolic blood pressure and the inverse of HDL-cholesterol. Multivariate linear regression analyses were used to quantify the associations between SED-time, MVPA,
\end{abstract}

Electronic supplementary material The online version of this article (doi:10.1007/s00125-013-3069-8) contains peer-reviewed but unedited supplementary material, which is available to authorised users.

A. J. M. Cooper $\cdot$ S. Brage $\cdot$ U. Ekelund $\cdot$ N. J. Wareham •

S. J. Griffin · R. K. Simmons $(\bowtie)$

MRC Epidemiology Unit, University of Cambridge, Institute of

Metabolic Science, Box 285, Addenbrooke's Hospital, Hills Road,

Cambridge CB2 0QQ, UK

e-mail: Rebecca.simmons@mrc-epid.cam.ac.uk

U. Ekelund

Department of Sport Medicine, Norwegian School of Sport Sciences,

Ullevål Stadion, Oslo, Norway

S. J. Griffin

Primary Care Unit, Institute of Public Health, University of

Cambridge, Cambridge, UK
PAEE and cardiorespiratory fitness with individual metabolic risk factors and clustered metabolic risk.

Results Each additional $1 \mathrm{~h}$ of SED-time was positively associated with clustered metabolic risk, independently of sleep duration and MVPA $(\beta=0.16[95 \%$ CI $0.03,0.29])$. After accounting for SED-time, MVPA was associated with systolic blood pressure $(\beta=-2.07[-4.03,-0.11])$ but not with clustered metabolic risk $(\beta=0.01[-0.28,0.30])$. PAEE and cardiorespiratory fitness were significantly and independently inversely associated with clustered metabolic risk $(\beta=-0.03[-0.05,-0.02]$ and $\beta=-0.06$ $[-0.10,-0.03]$, respectively). Associations between SED-time and metabolic risk were generally stronger in the low compared with the high fitness group.

Conclusions/interpretation PAEE was inversely associated with metabolic risk, whereas SED-time was positively associated with metabolic risk. MVPA was not associated with clustered metabolic risk after accounting for SED-time. Encouraging this high-risk group to decrease SED-time, particularly those with low cardiorespiratory fitness, and increase their overall physical activity may have beneficial effects on disease progression and reduction of cardiovascular risk.

Trial registration: ISRCTN99175498

Keywords ADDITION-Plus · Cardiovascular risk · Cohort . Physical activity $\cdot$ Sedentary $\cdot$ Type 2 diabetes

\begin{tabular}{|c|c|}
\hline \multicolumn{2}{|c|}{ Abbreviations } \\
\hline \multirow[t]{3}{*}{ ADDITION } & Anglo-Danish-Dutch Study of Intensive \\
\hline & Treatment in People with Screen Detected \\
\hline & Diabetes in Primary Care \\
\hline CVD & Cardiovascular disease \\
\hline IQR & Interquartile range \\
\hline MET value & Metabolic equivalent of task value \\
\hline MVPA & $\begin{array}{l}\text { Moderate-to-vigorous-intensity physical } \\
\text { activity }\end{array}$ \\
\hline
\end{tabular}




$\begin{array}{ll}\text { PA } & \text { Physical activity } \\ \text { PAEE } & \text { Physical activity energy expenditure } \\ \text { RMR } & \text { Resting metabolic rate } \\ \text { SED-time } & \text { Sedentary time } \\ \text { zMS } & \text { Standardised clustered metabolic risk score }\end{array}$

\section{Introduction}

Physical inactivity is an important modifiable lifestyle risk factor associated with hyperglycaemia, hypertension, dyslipidaemia and the risk of developing cardiovascular disease (CVD) in both healthy individuals and those with type 2 diabetes [1-5]. Despite the clear health benefits of physical activity (PA), however, individuals with type 2 diabetes generally have low levels of PA $[6,7]$ and have difficulty maintaining the required level necessary for improving metabolic health [8]. Emerging evidence suggests that targeting sedentary time (SED-time) may represent an additional approach to reducing metabolic risk in apparently healthy populations [9-11]. The assessment of PA and SED-time in these studies, however, has typically been derived from self-report measures. The imprecision and bias associated with selfreport measures means that their ability to accurately assess total activity, as well as PA intensity and SED-time, is limited. The single study using an objective measure of PA in individuals with type 2 diabetes showed that SED-time was positively associated with metabolic risk, whereas moderate-tovigorous-intensity physical activity (MVPA) was only weakly inversely associated with risk [12]. Given the limited amount of time spent in MVPA in this population, and the large amount of SED-time, targeting reductions in SED-time by increasing overall physical activity energy expenditure (PAEE), independently of MVPA, may offer a more feasible strategy to managing metabolic risk. Before advocating trials aimed at reducing SED-time in this population, however, it is important first to elucidate whether this association is independent of sleep duration, which might confound the association with metabolic risk [13]. As the association between different dimensions of PA and metabolic risk may differ by level of cardiorespiratory fitness [14], it is also important to establish whether these associations are modified by cardiorespiratory fitness.

Using data from the ADDITION-Plus trial of individuals with type 2 diabetes, which included objective measures of PA using a combined heart rate and movement sensor, we aimed to: (1) examine the magnitude, direction and relative associations between SED-time, MVPA, PAEE and cardiorespiratory fitness with metabolic risk; and (2) determine whether associations of SED-time, MVPA and PAEE differ by level of cardiorespiratory fitness.

\section{Methods}

The design and rationale for the ADDITION-Plus study have been reported previously [15]. In brief, ADDITION-Plus is a randomised controlled trial nested within the intensive treatment arm of the ADDITION-Cambridge study, which evaluated the efficacy of a facilitator-led, theory-based behaviour change intervention for recently diagnosed type 2 diabetic patients. Thirty-four general practices in East Anglia participated in the study. Eligible individuals were those aged 40-69 years who had been diagnosed with type 2 diabetes following screening in the ADDITION study or clinically diagnosed during the previous 3 years in participating general practice surgeries. Exclusion criteria were women who were pregnant or lactating or those with a likely survival prognosis of less than 1 year. Out of 1,109 eligible individuals, 478 agreed to participate in ADDITION-Plus and were individually randomised to receive either intensive treatment alone $(n=239)$ or intensive treatment plus the facilitator-led individual behaviour change intervention $(n=239)$. The two trial arms were pooled and a cohort analysis conducted. All participants gave written informed consent, and the study was approved by the Eastern Multi-Centre Research Ethics Committee (reference number 02/5/54). The trial is registered as ISRCTN99175498.

Assessment of PA, cardiorespiratory fitness, sleep duration and metabolic risk factors Assessment of ADDITION-Plus participants included physiological and anthropometric measures by trained staff following standard operating procedures, venesection and completion of self-report questionnaires. PA was assessed using a combined heart rate and movement sensor (Actiheart; CamNtech, Cambridge, UK) worn continuously for 4 days in $30 \mathrm{~s}$ resolution [16]. A graded treadmill walk test was used to calibrate heart rate individually [17] and to estimate cardiorespiratory fitness by extrapolation of the heart rate/oxygen consumption relationship to age-predicted maximal heart rate. For participants who did not complete an individual calibration test $(n=170)$, we used all valid calibration tests in the rest of the sample $(n=308)$ to derive a group calibration equation adjusted for age, sex, beta blocker $(y e s=94, n o=214)$ and sleeping heart rate for the translation of heart rate into activity intensity. Heart rate data collected during the free-living period were processed using noise classification followed by Gaussian robust regression [18], and average activity intensity $\left(\mathrm{J} \mathrm{min}^{-1} \mathrm{~kg}^{-1}\right)$ was estimated using a branched equation framework [19]. Resulting time-series data were summarised into PAEE $\left(\mathrm{kJ} \mathrm{kg}^{-1} \mathrm{day}^{-1}\right)$, SED-time and MVPA (min/day), whilst minimising diurnal information bias caused by non-wear periods (segments of non-physiological data). Cardiorespiratory fitness $\left(\dot{V} \mathrm{O}_{2 \text { max.pred }}\right)$ was assessed for 291 participants (196 men and 95 women) using extrapolation of heart rate response to a submaximal ramped exercise test to age-predicted maximum heart rate $(208-0.7 \times$ age $)$, as 
described elsewhere [17]. SED-time was defined as a metabolic equivalent of task value (MET value) of $<1.5$, in accordance with current convention [20], and MVPA as $\geq 3.0$ MET, both primarily using the Oxford estimate of resting metabolic rate (RMR) to define 1 MET [21], and secondarily using a fixed value of $20.35 \mathrm{~J} \mathrm{ml} \mathrm{O} \times 3.5 \mathrm{ml} \mathrm{O}_{2} \mathrm{~min}^{-1} \mathrm{~kg}^{-1}$. Sensitivity analyses were run to test the robustness of results with regard to MET thresholds ( $<1.75$ MET vs $<1.5$ MET for SEDtime, and $\geq 4.0$ MET vs $\geq 3.0$ MET for MVPA). We overlaid self-reported sleep timings on the objective time-series data, the appropriateness of which was verified by visual inspection (see Methods, Covariate assessment, for a full explanation). From these data, we calculated sleep duration and non-sleep SED-time.

Blood pressure was calculated as the mean of three measurements performed after $10 \mathrm{~min}$ of rest and with participants seated with a cuff placed on the predominant arm at the level of the heart, using an automatic sphygmomanometer (Omron M4; Milton Keynes, UK). Body weight and height were measured in light clothing and without shoes using a scale (SECA; Birmingham, UK) and a fixed rigid stadiometer, respectively. Waist circumference was calculated as the average of two measurements taken halfway between the lowest point of the rib cage and the anterior superior iliac crest while standing. $\mathrm{HbA}_{1 \mathrm{c}}$ was measured in venous samples using an ion-exchange high-performance liquid chromatography method (Tosoh Bioscience, Redditch, UK). Serum total cholesterol, HDL-cholesterol and triacylglycerol were measured using enzymatic techniques (Dade Behring Dimension Analyzer; Dade Behring, Newark, NJ, USA). Standardised selfreport questionnaires were used to collect information on sociodemographic characteristics and sleep duration.

Covariate assessment Medication adherence was assessed by the Medication Adherence Report Schedule (MARS) questionnaire [22]. Smoking status was assessed by questionnaire with a yes/no answer to being a: current smoker, former smoker or never smoker. Dietary intake was evaluated using a validated food frequency questionnaire [23]. Occupational socioeconomic class was evaluated by questionnaire and was subsequently collapsed into three classes: (1) higher managerial, administrative and professional occupations; (2) intermediate occupations; and (3) routine and manual occupations [24]. Habitual bedtime and wake time were self-reported for weekdays and weekends using the EPIC-Norfolk Physical Activity Questionnaire (EPAQ2) [25]. Sleep duration was calculated as $(5 / 7 \times$ weekday sleep duration $)+(2 / 7 \times$ weekend sleep duration). We overlaid the self-reported sleep timings on the time-series data from the combined heart rate and movement sensor to visually identify participants who had high motion/heart rate periods (which we identified as being indistinguishable from time spent awake and in contrast to combined heart rate and movement sensor data during time spent sleeping) exceeding their self-reported bedtimes and/or wake times by $\geq 30 \mathrm{~min}$. Agreement between the self-reported bedtimes and wake times with the objective time-series data was assessed by two examiners independently for each participant (A. J. M. Cooper and K. Westgate [Medical Research Council Epidemiology Unit, Cambridge, UK]). The kappa statistic for agreement between examiners was 0.77. Disagreement between examiners was resolved by re-examination of the data and discussion. In total, self-reported bedtimes and wake times were deemed to be incompatible with the objective time-series data for 21 participants, who were excluded from analyses.

Calculation of the clustered metabolic risk $z$ score A summary score of clustered metabolic risk (zMS) was calculated by summing standardised values for waist circumference, fasting triacylglycerol, $\mathrm{HbA}_{1 \mathrm{c}}$ and systolic blood pressure and the inverse of HDL-cholesterol. Variables were standardised by subtracting the sample mean from the individual mean and dividing by the SD.

Complete data on objectively measured PA, metabolic risk factors and potential confounding variables were available for 394 participants ( $n=291$ for cardiorespiratory fitness).

Statistical analysis Descriptive characteristics were summarised separately for men and women using means with SDs, medians with interquartile ranges (IQR) or frequencies. Wilcoxon rank-sum tests, $t$ tests or $\chi^{2}$ tests were used to examine whether there were any differences in participant characteristics between those with and those without missing data. Fasting triacylglycerol values were log transformed (base $e$ ) due to their non-normal distribution. Associations between SEDtime, MVPA, PAEE, $\dot{V} \mathrm{O}_{2 \text { max.pred }}$ and sleep duration were estimated using Pearson correlation coefficients.

We used multivariate linear regression analyses to model the associations between SED-time (h/day), MVPA (h/day), PAEE $\left(\mathrm{kJ} \mathrm{kg}^{-1}\right.$ day $\left.^{-1}\right)$ and $\dot{V} \mathrm{O}_{2 \text { max.pred }}\left(\mathrm{ml} \mathrm{O}_{2} \mathrm{~kg}^{-1} \mathrm{~min}^{-1}\right)$ with individual subcomponents of the zMS (waist circumference, fasting triacylglycerol, $\mathrm{HbA}_{1 \mathrm{c}}$, systolic blood pressure and inverted HDL-cholesterol), and with the clustered metabolic risk score as one variable after confirming that our data met the assumptions underlying linear regression (i.e. linearity, normality, homoscedasticity and absence of multicolinearity). To compare directly the relative contribution of SED-time, MVPA, PAEE and $\dot{V} \mathrm{O}_{2 \text { max.pred }}$ we also expressed these exposure variables in the same units (per SD difference). Under the assumption that these variables are measured with the same degree of measurement error, their expression in standardised form allows direct comparisons of the magnitude of association between measures and clustered metabolic risk. All regression models are presented unadjusted (crude) and adjusted for age, sex, intervention group, occupational socioeconomic class, smoking status, 
sleep duration, total energy intake, percentage of energy from fat, alcohol intake and waist circumference (except when waist circumference or zMS were modelled as the outcome). When the outcome of interest was blood pressure, $\mathrm{HbA}_{1 \mathrm{c}}$, triacylglycerol or HDL-cholesterol, we additionally adjusted for the use of antihypertensive, glucose-lowering or lipidlowering medication, respectively. For the zMS we adjusted for the use of antihypertensive, glucose-lowering and lipidlowering medications. Finally, to examine the association between SED-time (independently of MVPA) and MVPA (independently of SED-time) with subcomponents of the zMS and the score as one variable, we additionally adjusted for MVPA and SED-time, respectively.

All data were analysed in continuous form, although some data were categorised into tertiles for illustrative purposes. Finally, we examined whether the associations with SEDtime, MVPA, PAEE and $\dot{V} \mathrm{O}_{2 \text { max.pred }}$ with zMS were modified by age ( $<60$ vs $\geq 60$ years) and sex. We also examined whether the associations of SED-time, MVPA and PAEE with metabolic risk factors and clustered metabolic risk were modified by cardiorespiratory fitness levels (continuous). In further sensitivity analyses we examined whether our results would have differed by excluding participants with fewer than 3 days of combined heart rate and movement sensor data $(n=21)$, or by excluding participants who did not complete an individual calibration test $(n=103)$.

All statistical analyses were performed using Stata/SE 12.1 (StataCorp LP, College Station, TX, USA).

\section{Results}

Table 1 shows the anthropometric and metabolic characteristics of participants with complete data at 1 year $(n=394)$, stratified by sex. The mean (SD) age of men and women was 60.2 (7.4) and 60.5 (7.4) years, respectively. More men than women met the inclusion criteria and agreed to participate in the study. Participants with missing data $(n=84)$ had similar baseline values for all anthropometric and metabolic characteristics shown in Table 1 (data not shown). As expected, men were taller and heavier than women, had a larger waist circumference and reported consuming a greater amount of alcohol. Men had higher systolic blood pressure and triacylglycerol levels compared with women, whereas women had higher total and HDL-cholesterol levels. Total PAEE was higher among men than among women (37.6 vs $29.6 \mathrm{~kJ} \mathrm{~kg}^{-1}$ day $^{-1}$, respectively; $p<0.001$ ) and men had higher levels of cardiorespiratory fitness than women (33.4 $\mathrm{ml} \mathrm{O}_{2} \mathrm{~kg}^{-1} \mathrm{~min}^{-1}$ vs $25.2 \mathrm{ml} \mathrm{O}_{2} \mathrm{~kg}^{-1} \mathrm{~min}^{-1}$, respectively; $p<0.001$ ). Men and women had similar amounts of SEDtime ( $9.1 \mathrm{vs} 9.0 \mathrm{~h} /$ day, respectively; $p=0.69$ ), but men reported spending fewer hours sleeping $(8.1$ vs $8.7 \mathrm{~h} /$ day, respectively; $p<0.001$ ) and were more likely than women to spend time in MVPA $(p=0.007)$. SED-time was strongly inversely correlated with time spent in MVPA $(r=-0.60$; $p<0.001)$ and with PAEE $(r=-0.76 ; p<0.001)$. SED-time was weakly inversely correlated with sleep duration $(r=-0.28$; $p<0.001)$. Cardiorespiratory fitness was moderately correlated with MVPA $(r=0.44 ; p<0.001)$ and PAEE $(r=0.49 ; p<0.001)$, and weakly inversely correlated with SED-time $(r=-0.11$; $p<0.001)$. MVPA was strongly correlated with PAEE $(r=0.90 ; p<0.001)$.

Table 2 shows crude and adjusted associations between SED-time (h/day), MVPA (h/day), PAEE ( $\mathrm{kJ} \mathrm{kg}^{-1} \mathrm{day}^{-1}$ ) and $\dot{V} \mathrm{O}_{2 \text { max.pred }}$ with subcomponents of the zMS and the clustered metabolic risk score as one variable. In adjusted analyses, PAEE was inversely associated with waist circumference $(\beta=-0.27 \mathrm{~cm} ; 95 \% \mathrm{CI}-0.35,-0.20)$ and clustered metabolic risk ( $\beta=-0.03 ; 95 \% \mathrm{CI}-0.05,-0.02)$. Results were similar for $\dot{V} \mathrm{O}_{2 \text { max.pred }}$ (Table 2). By contrast, SED-time was positively associated with waist circumference $(\beta=0.68 \mathrm{~cm}$; $95 \%$ CI $0.01,1.35)$, triacylglycerol levels $(\beta=0.03 ; 95 \%$ CI $0.00,0.06)$ and clustered metabolic risk $(\beta=0.16 ; 95 \% \mathrm{CI}$ $0.03,0.29)$, and inversely associated with HDL-cholesterol levels $(\beta=-0.02 ; 95 \% \mathrm{CI}-0.03,-0.001)$ in analyses adjusted for time spent in MVPA. After accounting for SED-time, time spent in MVPA only remained significantly inversely associated with systolic blood pressure $(\beta=-2.07 ; 95 \%$ CI -4.03 , -0.11 ). The bottom row of Table 2 shows crude and adjusted associations between standardised measures (SDs) of PAEE, SED-time and MVPA with clustered metabolic risk. The magnitude of the association between PAEE and metabolic risk was similar to the magnitude between SED-time and metabolic risk, although in the opposite direction, such that each SD increase in PAEE was associated with a lower metabolic risk ( $\beta=-0.55 ; 95 \% \mathrm{CI}-0.81,-0.28)$, whereas each SD increase in SED-time was associated with a higher metabolic risk ( $\beta=0.42 ; 95 \%$ CI $0.09,0.75)$. Figure 1 highlights the association of SED-time, MVPA, PAEE and $\dot{V} \mathrm{O}_{2 \text { max.pred }}$ with metabolic risk after adjustment for potential confounding factors. Table 1 in the electronic supplementary material (ESM) shows the associations between metabolic risk variables with SED-time and MVPA using a fixed value of RMR to define 1 MET; these results were largely similar to those reported using individualised RMR, except for a stronger inverse association between MVPA and waist circumference, which remained significant after adjustment for SED-time.

There was no evidence to suggest that the associations between SED-time, MVPA, PAEE and $\dot{V}_{2 \text { max.pred }}$ with zMS were modified by either age (all $p$ values $\geq 0.88$ ) or sex (all $p$ values $\geq 0.32$ ). Although the associations of SED-time, MVPA and PAEE with metabolic risk factors and clustered metabolic risk did not differ by level of cardiorespiratory fitness (all $p$ values for interaction $\geq 0.07$, except for the association between PAEE and $\mathrm{HbA}_{1 \mathrm{c}}[p=0.03]$ ), our results 
Table 1 Anthropometric and metabolic characteristics of ADDITION-Plus participants $(n=394)$, stratified by sex

\begin{tabular}{|c|c|c|c|}
\hline Characteristic & $\operatorname{Men}(n=250)$ & Women $(n=144)$ & $\begin{array}{l}p \text { value for difference } \\
\text { between groups }\end{array}$ \\
\hline Age, years & $60.2(7.4)$ & $60.5(7.4)$ & 0.71 \\
\hline Occupational socioeconomic class, $\%$ & & & 0.01 \\
\hline Managerial & 47.2 & 31.9 & \\
\hline Intermediate & 22.0 & 30.6 & \\
\hline Routine and manual & 30.8 & 37.5 & \\
\hline Height, $\mathrm{cm}$ & $174.9(7.2)$ & $161.4(6.9)$ & $<0.001$ \\
\hline Weight, kg & $96.9(16.7)$ & $85.9(17.7)$ & $<0.001$ \\
\hline BMI, $\mathrm{kg} / \mathrm{m}^{2}$ & $31.6(5.1)$ & $32.9(6.0)$ & 0.03 \\
\hline Waist circumference, $\mathrm{cm}$ & $111.3(12.5)$ & $105.0(13.0)$ & $<0.001$ \\
\hline Systolic blood pressure, $\mathrm{mmHg}$ & $132.0(16.7)$ & $125.9(17.0)$ & $<0.001$ \\
\hline Triacylglycerol, mmol// ${ }^{\mathrm{a}}$ & $1.7(1.2-2.3)$ & $1.6(1.1-2.1)$ & 0.06 \\
\hline Total cholesterol, mmol/1 & $4.2(0.9)$ & $4.4(0.8)$ & 0.008 \\
\hline HDL-cholesterol, mmol/1 & $1.1(0.3)$ & $1.3(0.3)$ & $<0.001$ \\
\hline $\mathrm{HbA}_{1 \mathrm{c},} \%$ & $6.7(1.0)$ & $6.6(0.8)$ & 0.68 \\
\hline $\mathrm{HbA}_{1 \mathrm{c}}, \mathrm{mmol} / \mathrm{mol}$ & 49.7 & 48.6 & \\
\hline On glucose-lowering medication, $\%$ & 49.6 & 54.9 & 0.31 \\
\hline On antihypertensive medication, $\%$ & 72.4 & 70.1 & 0.63 \\
\hline On lipid-lowering medication, $\%$ & 74.0 & 79.9 & 0.19 \\
\hline Total energy intake, $\mathrm{kJ} /$ day $^{\mathrm{a}}$ & $7,272(6,130,8,489)$ & $6,602(5,711,8,489)$ & 0.06 \\
\hline Percentage of energy from fat, $\%$ & $30.6(5.8)$ & $30.9(5.7)$ & 0.62 \\
\hline Alcohol intake, $\mathrm{g} /$ day $^{\mathrm{a}}$ & $6.0(1.0,15.0)$ & $2.0(0.0,5.0)$ & $<0.001$ \\
\hline PAEE, $\mathrm{kJ} \mathrm{kg}^{-1}$ day $^{-1} \mathrm{~b}$ & $37.6(18.2)$ & $29.6(13.5)$ & $<0.001$ \\
\hline Average sleep duration, $\mathrm{h} /$ day & $8.1(1.1)$ & $8.7(0.9)$ & $<0.001$ \\
\hline Duration SED-time, $\mathrm{h} / \mathrm{day}^{\mathrm{b}, \mathrm{c}}$ & $9.1(2.7)$ & $9.0(2.4)$ & 0.69 \\
\hline Duration SED-time, h/day ${ }^{\mathrm{b}, \mathrm{d}}$ & $10.3(2.6)$ & $10.5(2.2)$ & 0.50 \\
\hline Duration MVPA, h/day ${ }^{\mathrm{b}, \mathrm{c}}$ & $1.4(1.2)$ & $1.1(0.9)$ & 0.007 \\
\hline Duration MVPA, h/day ${ }^{\mathrm{b}, \mathrm{d}}$ & $0.88(0.92)$ & $0.43(0.48)$ & $<0.001$ \\
\hline$\dot{V} \mathrm{O}_{2 \text { max.pred }}, \mathrm{ml} \mathrm{O}_{2} \mathrm{~kg}^{-1} \min ^{-1 \mathrm{a}, \mathrm{e}}$ & $33.4(28.1,37.4)$ & $25.2(20.2,31.9)$ & $<0.001$ \\
\hline
\end{tabular}

Data are means (SD) unless stated otherwise

${ }^{\mathrm{a}}$ Median (IQR)

${ }^{\mathrm{b}}$ Individually calibrated where available $(n=291)$, otherwise group calibrated $(n=103)$

${ }^{c} 1$ MET defined using individual estimate of RMR [21]

d 1 MET defined using $71.2 \mathrm{~J} \mathrm{~min}^{-1} \mathrm{~kg}^{-1}$

${ }^{\text {e }} \dot{V} \mathrm{O}_{2 \text { max.pred }}$ available for 291 participants (196 men and 95 women)

were suggestive of a stronger association between SED-time and subcomponents of metabolic risk among individuals below the median for cardiorespiratory fitness (ESM Table 2). MVPA was strongly inversely associated with systolic blood pressure among the least fit $(\beta=-4.85 \mathrm{mmHg} ; 95 \% \mathrm{CI}-9.22$, -0.48 ). The estimated $\beta$ coefficients and $95 \%$ CIs were not significantly different between models if we excluded participants with fewer than 3 days of combined heart rate and movement sensor data $(n=21)$ (all $p$ values $\geq 0.36)$ or we excluded participants who did not complete an individual calibration test $(n=103)$ (all $p$ values $\geq 0.46)$. Although our results remained robust with regard to changes in MET thresholds for SED-time, a threshold for MVPA of $\geq 4.0$ METs was more strongly inversely associated (numerically larger $\beta$ coefficient), albeit non-significantly, with waist circumference, systolic blood pressure and clustered metabolic risk compared with an MVPA threshold of $\geq 3.0$ METs (ESM Table 3).

\section{Discussion}

In this cross-sectional study of individuals with recently diagnosed type 2 diabetes, higher levels of SED-time were 


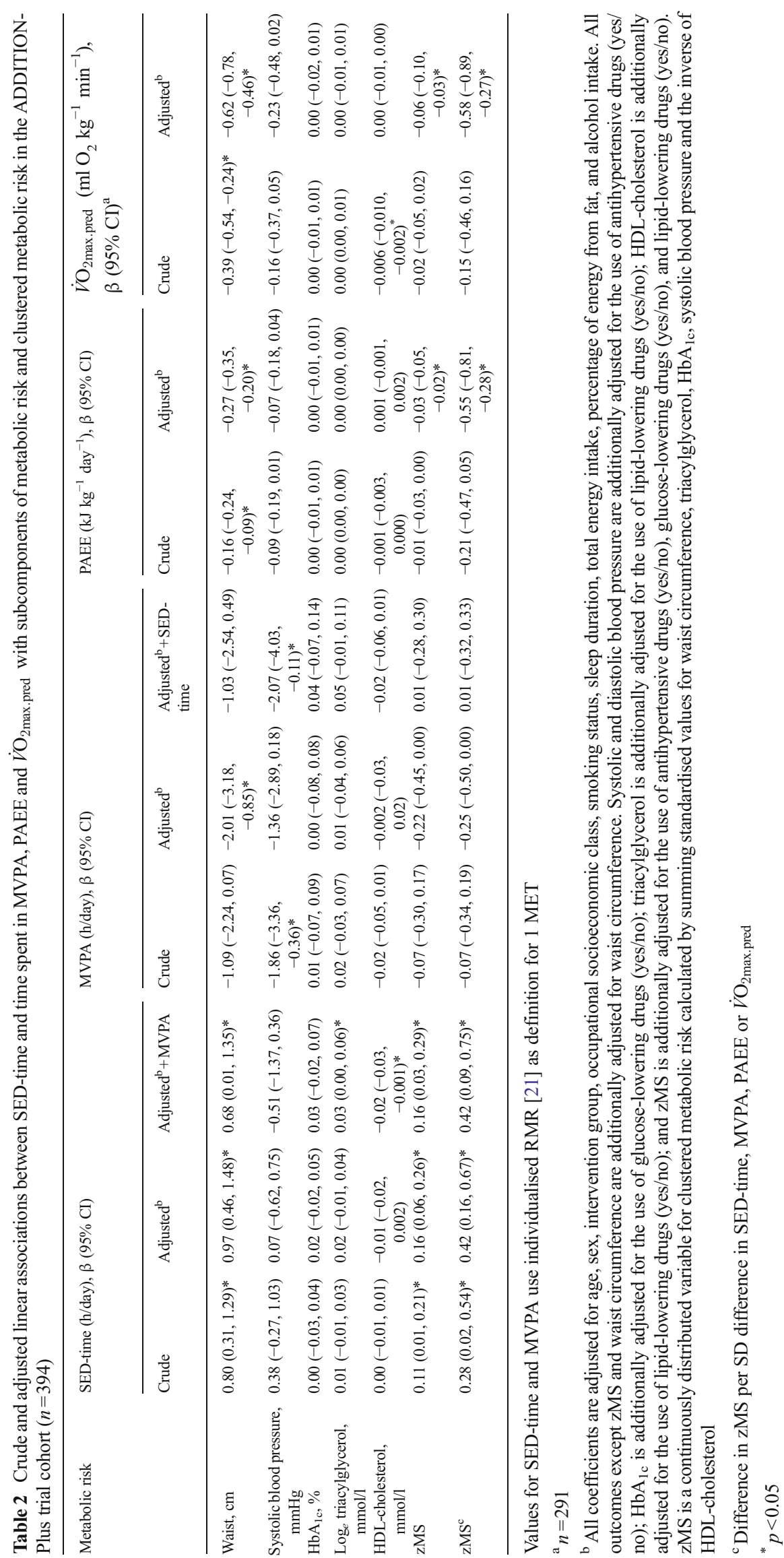


a

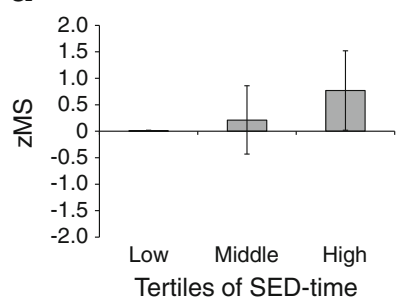

C

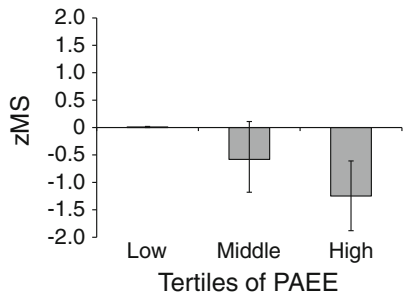

b

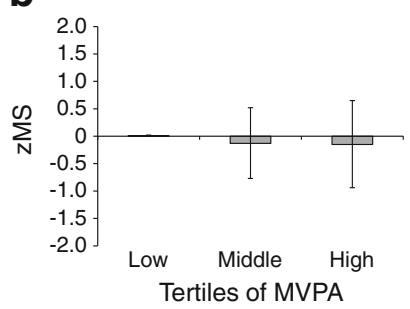

d

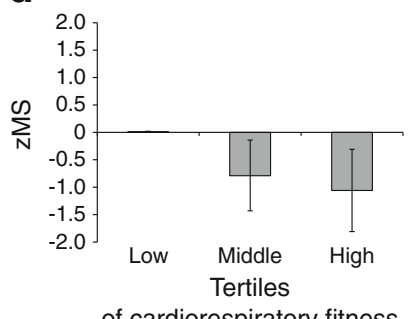

of cardiorespiratory fitness

Fig. 1 Associations of SED-time, MVPA, PAEE and cardiorespiratory fitness with clustered metabolic risk. Mean SED-time in the lower (reference), middle and higher tertile groups was 6.4 (range 1.2-8.0), 9.1 (range 8.0-10.4) and 11.6 (range 10.4-16.4) h/day, respectively (a). Mean time spent in MVPA in the lower, middle and higher tertile groups was 0.4 (range 0.0-0.6), 1.0 (range 0.6-1.5) and 2.4 (range 1.5-6.1) h/day, respectively (b). Mean PAEE in the lower, middle and higher tertile groups was 18.9 (range 5.6-24.7), 31.2 (range 24.8-39.4) and 50.3 (range 39.6-98.8) $\mathrm{kJ} \mathrm{kg}^{-1}$ day ${ }^{-1}$, respectively (c). Mean cardiorespiratory fitness in the lower (reference), middle and higher tertile groups was 21.0 (range 11.3-26.9), 31.0 (range 27.1-34.6) and 38.7 (range 34.8-58.6) $\mathrm{ml} \mathrm{O}_{2} \mathrm{~kg}^{-1} \mathrm{~min}^{-1}$, respectively (d). Data are the zMS for each group $(95 \% \mathrm{CI})$, adjusted for age, sex, intervention group, occupational socioeconomic class, smoking status, sleep duration, total energy intake, percentage energy from fat, alcohol intake, and use of antihypertensive drugs, glucose-lowering drugs and lipid-lowering drugs. SED-time is additionally adjusted for MVPA, and MVPA is additionally adjusted for SED-time. $p$ values for trend across categories for SED-time (a), MVPA (b), PAEE (c) and cardiorespiratory fitness (d) were $p=0.04, p=0.72$, $p<0.001$ and $p=0.001$, respectively

associated with higher levels of metabolic risk, independently of measured confounders and time spent in MVPA. In contrast, time spent in MVPA was not significantly associated with any metabolic risk factor except for systolic blood pressure and waist circumference after accounting for measured confounders and SEDtime. Total PAEE and $\dot{V} \mathrm{O}_{2 \text { max.pred }}$ were inversely associated with waist circumference and a lower metabolic risk profile, independently of measured confounders. Associations between SED-time and measures of metabolic risk were stronger in those with low fitness levels compared with those in the high fitness group, suggesting that higher levels of cardiorespiratory fitness may provide some protection against the deleterious effects of SED-time. Our findings suggest that decreasing the amount of SED-time and increasing overall PA may have beneficial effects on disease progression and cardiovascular risk in patients with type 2 diabetes.

Our study exhibits several strengths, including the use of standardised measures and objective measurement of PA and SED-time. The few previous studies that have objectively assessed SED-time and/or PA have used either heart rate or movement sensing [12, 26-28]. Deriving PA from heart rate measurements alone can lead to imprecision due to the difficulty in distinguishing time spent resting from time spent engaging in low levels of PA that do not result in elevations in heart rate. By contrast, relying on movement sensing alone is limited due to the fact that acceleration of typically only one body segment is recorded, and as such certain activities will not be registered (e.g. cycling). Using a combined heart rate and movement sensor allowed us to discriminate between both rest and low-intensity locomotor activities across biomechanically different activities. This is important in a study population that spends a considerable amount of time sedentary and very little time in MVPA. Our findings are unlikely to be biased as participants with and without missing data were similar. Finally, by using objective time-series data for sleep duration, verified by overlaying self-reported sleep time, we were able to examine all associations independently of sleep, which cannot be considered the same as SED-time in terms of metabolic risk $[29,30]$.

Some potential limitations of our study merit discussion. First, as this is a cross-sectional study we cannot infer the direction or the causal nature of the associations. Nevertheless, findings from animal models have shown that a lack of local contractile muscle stimulation, such as when sedentary, can adversely affect triacylglycerol, HDL-cholesterol and other metabolic risk factors as a result of suppression of muscle lipoprotein lipase stimulation [31]. Second, despite the transient increase in heart rate when changing posture from sitting to standing [32,33], it is difficult to accurately distinguish between time spent sitting and time spent standing using the combined heart rate and movement data acquired in this study, and as such it is possible that time spent standing was misclassified as SED-time and vice versa. Any potential misclassification of standing time as SED-time, however, would likely have led to attenuation of the associations with SED-time. Similarly, the questionnaire used to collect data on habitual bedtime and wake time in this study did not include questions related to naps or an estimate of actual sleep duration, which might have resulted in sleep time being misclassified as SED-time and vice versa. Use of a more precise measure of sleep-related variables, such as polysomnography, might reduce misclassification, but these measures are currently not feasible in epidemiological studies. Third, beneficial associations of MVPA with metabolic risk factors might have been missed as a result of the definition of MVPA, as suggested by the stronger, albeit non-significant, inverse associations with waist circumference, systolic blood pressure and clustered metabolic risk when we used a more 
stringent cut point of $\geq 4.0 \mathrm{METs}$, as opposed to $\geq 3.0 \mathrm{METs}$. Associations with MVPA were not materially different when we used a fixed intensity threshold of $142.5 \mathrm{~J} \mathrm{~min}^{-1} \mathrm{~kg}^{-1}$ above rest ( 3 METs) to define MVPA, rather than an individualised threshold, except for a stronger inverse association with waist circumference, which remained after adjustment for SED-time. Fourthly, cardiorespiratory fitness was not measured directly with a maximal test but was assessed using extrapolation of heart rate response to a submaximal ramped exercise test to age-predicted maximum heart rate. Finally, we cannot exclude the possibility of residual confounding or confounding by unmeasured factors.

Previous cross-sectional and prospective studies have shown beneficial associations between high levels of objectively assessed total PA with HDL-cholesterol and triacylglycerol, insulin and glucose levels in healthy individuals, while longer durations of SED-time have been adversely associated with waist circumference, insulin levels, triacylglycerol levels and overall metabolic risk [26, 28, 34-37]. Further, measures of glucose homeostasis have also been shown to be detrimentally associated with SED-time among individuals with newly diagnosed type 2 diabetes [12]. However, when the association between 6-month change in SED-time and metabolic risk was examined prospectively, no association with glucose homeostasis was observed [12]. Differences between crosssectional and prospective findings may be explained by the fact that the sample size of existing prospective studies may be inadequate to detect small but biologically important associations related to changes in these behaviours, especially given the short duration of follow-up. Nevertheless, results of studies with objective measures of PAEE and SED-time are consistent with those of prospective studies which have used selfreported data and have shown that PA is a good predictor of CVD morbidity and mortality [38-41] and that SED-time is associated with an increased risk of CVD events [4, 9]. The association between different dimensions of PA and metabolic risk has been shown to differ by cardiorespiratory fitness in healthy adults free from type 2 diabetes [14]. While our findings suggest that reductions in SED-time may be particularly beneficial among the least fit, they highlight the importance of reducing SED-time and increasing total PA for all patients with type 2 diabetes. The present results extend previous observations by using individually calibrated combined heart rate and movement sensing, which has been shown to have high validity for estimating PAEE in both laboratory [42] and free-living conditions [43] and which overcomes many of the limitations associated with either heart rate or movement monitoring alone [16]. Further, we demonstrate that the associations between PAEE and SED-time with metabolic risk are independent of sleep duration.

It is important to consider the clinical implications of the observed association between SED-time and the metabolic risk factors. Each $1 \mathrm{~h} /$ day increase in SED-time was associated with a $0.68 \mathrm{~cm}$ higher waist circumference, a $0.03 \mathrm{mmol} / \mathrm{l}$ higher triacylglycerol level and a $0.02 \mathrm{mmol} / \mathrm{l}$ lower HDLcholesterol level. The difference in SED-time between the 25th and 75th percentiles, however, was almost $4 \mathrm{~h} /$ day, which suggests that if the association were causal then it is not unreasonable to suggest that moving from the top to the bottom quartile of SED-time could result in a reduction in waist circumference of over $2.5 \mathrm{~cm}$, a reduction in triacylglycerol levels of $0.12 \mathrm{mmol} / \mathrm{l}$ and an increase in HDL-cholesterol by $0.08 \mathrm{mmol} / \mathrm{l}$. In context, changes of this magnitude have each been associated with a 3-5\% reduction in CVD risk [44-46].

Our findings support the hypothesis that the biological responses to SED-time likely influence metabolic risk through pathways distinct from those which have been implicated for MVPA [47, 48]. Our data also suggest that while adults with type 2 diabetes may gain additional health benefits from participating in MVPA, reducing SED-time and increasing overall activity, even if it is less than the recommended guidelines [49] it will result in important health benefits. Thus, our findings are consistent with the recommendations of the American Diabetes Association [1], highlighting the importance not only of MVPA but also of increasing overall PA at the expense of SED-time. These findings highlight the need to develop interventions to reduce SED-time that might be incorporated into educational programmes for newly diagnosed patients. This will require better information about determinants of SED-time in this group, but in the meantime simple and cheap approaches such as the provision of pedometers might be recommended [50].

In conclusion, total PAEE, but not MVPA, was associated with improved metabolic risk status in this cohort of recently diagnosed type 2 diabetic patients. Individuals with higher levels of SED-time had higher levels of metabolic risk, and this was particularly noticeable among those with low cardiorespiratory fitness levels. Encouraging this high-risk group to decrease their SED-time by increasing overall PA may have beneficial effects on disease progression and reduction of CVD risk. Future research is needed in order to establish the prospective associations between changes in PAEE, SED-time and time spent in MVPA with metabolic risk, as well as to establish the determinants and maintenance of change in this population.

Acknowledgements We are grateful to all participants and the practices teams for taking part in the ADDITION-Plus trial: Arbury Road Surgery, Ashwell Surgery, Birchwood Surgery, Bottisham Medical Practice, Brookfields/Cherry Hinton, Buckden Surgery, Clarkson Surgery, Cornerstone Practice, Cornford House Surgery, Cottenham Surgery, Dr Eaton \& Partners (Saffron Walden), George Clare Surgery, Great Staughton Surgery, Haddenham Surgery, Hilton House Surgery, Manea Surgery, Milton Surgery, New Roysia Surgery, Orchard House Surgery, Orton Medical Practice, Lensfield Road Surgery, Parkhall Road Surgery, Park Medical Centre, Petersfield Medical Practice, Riverside Practice, Rookery Medical Centre, Rosalind Franklin House, South Street Surgery, St Mary's Surgery, Thaxted Surgery, The Old Exchange, The Spinney Surgery, The Surgery (Over) and Woolpit Surgery. 
We thank K. Westgate (Medical Research Council Epidemiology Unit, Cambridge, UK) for independently examining agreement between the self-reported bedtimes and wake times with the objective time-series data for each participant in order to enable calculation of sleep duration and non-sleep SED-time.

We thank the Cambridge University Hospitals National Health Service (NHS) Foundation Trust Department of Clinical Biochemistry and the National Institute for Health Research (NIHR) Cambridge Biomedical Research Centre, Core Biochemical Assay Laboratory, for carrying out the biochemical assays.

The ADDITION- Plus team includes: R. Amin, G. Baker, R. Barling, M. Betts, A. Dickinson, J. B. Echouffo Tcheugui, U. Ekelund, F. Finucane, S. Mayle, J. Mitchell, P. Roberts, L. Sargeant, M. Sims, K. Westgate, F. Whittle and the Field Epidemiology, Data Management, IT, Physical Activity, Technical and Study Coordination teams (Medical Research Council [MRC] Epidemiology Unit, Cambridge, UK); J. Argles, R. Bale, R. Barling, S. Boase, J. Brimicombe, R. Butler, T. Fanshawe, P. Gash, J. Grant, S. Griffin, W. Hardeman, I. Hobbis, A. L. Kinmonth, T. McGonigle, N. Popplewell, A. T. Prevost, J. Smith, M. Smith, S. Sutton, F. Whittle and K. Williams (General Practice and Primary Care Research Unit, University of Cambridge, Cambridge, UK). The General Practice and Primary Care Research Unit at the University of Cambridge (GP) and the MRC Epidemiology Unit in Cambridge jointly coordinated the baseline and 1 year follow-up phases of the study.

Funding The trial is supported by the Wellcome Trust, the MRC, Diabetes UK and NHS R\&D support funding. SJG was a member of the NIHR School for Primary Care Research. The General Practice and Primary Care Research Unit is supported by NIHR research funds. SJG receives support from the Department of Health NIHR programme grant funding scheme [RP-PG-0606-1259]. The views expressed in this publication are those of the authors and not necessarily those of the Department of Health.

Access to research materials Data are not currently available for public release.

Duality of interest The authors declare that there is no duality of interest associated with this manuscript.

Contribution statement AJMC had full access to all the data in the study and takes responsibility for the accuracy of the data analysis. SJG is a principal investigator for the ADDITION-Plus trial. AJMC, SJG, SB and RKS are the guarantors of this work and, as such, had full access to all data in the study and take responsibility for the integrity of the data and the accuracy of the data analysis. SJG and RKS acquired the data. AJMC, RKS and SJG conceived and designed the study question. AJMC and SB analysed the data, and AJMC, SB, UE, NJW, SJG and RKS interpreted the data. AJMC drafted the manuscript, and all authors critically revised the manuscript for important intellectual content and have approved the final version.

Open Access This article is distributed under the terms of the Creative Commons Attribution License which permits any use, distribution, and reproduction in any medium, provided the original author(s) and the source are credited.

\section{References}

1. Colberg SR, Sigal RJ, Fernhall B et al (2010) Exercise and type 2 diabetes: the American College of Sports Medicine and the American Diabetes Association: joint position statement. Diabetes Care 33: e147-e167
2. Kesaniemi YK, Danforth E Jr, Jensen MD, Kopelman PG, Lefebvre P, Reeder BA (2001) Dose-response issues concerning physical activity and health: an evidence-based symposium. Med Sci Sports Exerc 33:S351-S358

3. Ford ES, Caspersen CJ (2012) Sedentary behaviour and cardiovascular disease: a review of prospective studies. Int J Epidemiol 41:1338-1353

4. Wijndaele $\mathrm{K}$, Brage $\mathrm{S}$, Besson $\mathrm{H}$ et al (2011) Television viewing and incident cardiovascular disease: prospective associations and mediation analysis in the EPIC Norfolk Study. PloS ONE 6:e20058

5. Dunstan DW, Barr EL, Healy GN et al (2010) Television viewing time and mortality: the Australian Diabetes, Obesity and Lifestyle Study (AusDiab). Circulation 121:384-391

6. Jakicic JM, Gregg E, Knowler W et al (2010) Activity patterns of obese adults with type 2 diabetes in the Look AHEAD study. Med Sci Sports Exerc 42:1995-2005

7. Morrato EH, Hill JO, Wyatt HR, Ghushchyan V, Sullivan PW (2007) Physical activity in U.S. adults with diabetes and at risk for developing diabetes, 2003. Diabetes Care 30:203-209

8. Dunstan DW, Daly RM, Owen N et al (2005) Home-based resistance training is not sufficient to maintain improved glycemic control following supervised training in older individuals with type 2 diabetes. Diabetes Care 28:3-9

9. Wijndaele K, Healy GN, Dunstan DW et al (2010) Increased cardiometabolic risk is associated with increased TV viewing time. Med Sci Sports Exerc 42:1511-1518

10. Dunstan DW, Salmon J, Healy GN et al (2007) Association of television viewing with fasting and 2-h postchallenge plasma glucose levels in adults without diagnosed diabetes. Diabetes Care 30:516-522

11. Kronenberg F, Pereira MA, Schmitz MK et al (2000) Influence of leisure time physical activity and television watching on atherosclerosis risk factors in the NHLBI Family Heart Study. Atherosclerosis 153:433-443

12. Cooper AR, Sebire S, Montgomery AA et al (2012) Sedentary time, breaks in sedentary time and metabolic variables in people with newly diagnosed type 2 diabetes. Diabetologia 55:589-599

13. Knutson KL (2010) Sleep duration and cardiometabolic risk: a review of the epidemiologic evidence. Best Pract Res Clin Endocrinol Metab 24:731-743

14. Franks PW, Ekelund U, Brage S, Wong MY, Wareham NJ (2004) Does the association of habitual physical activity with the metabolic syndrome differ by level of cardiorespiratory fitness? Diabetes Care 27:1187-1193

15. Griffin SJ, Simmons RK, Williams KM et al (2011) Protocol for the ADDITION-Plus study: a randomised controlled trial of an individually-tailored behaviour change intervention among people with recently diagnosed type 2 diabetes under intensive UK general practice care. BMC Public Health 11:211

16. Brage S, Brage N, Franks PW, Ekelund U, Wareham NJ (2005) Reliability and validity of the combined heart rate and movement sensor Actiheart. Eur J Clin Nutr 59:561-570

17. Brage S, Ekelund U, Brage N et al (2007) Hierarchy of individual calibration levels for heart rate and accelerometry to measure physical activity. J Appl Physiol 103:682-692

18. Stegle O, Fallert SV, MacKay DJ, Brage S (2008) Gaussian process robust regression for noisy heart rate data. IEEE Trans Biomed Eng $55: 2143-2151$

19. Brage S, Brage N, Franks PW et al (2004) Branched equation modeling of simultaneous accelerometry and heart rate monitoring improves estimate of directly measured physical activity energy expenditure. J Appl Physiol 96:343-351

20. Sedentary Behaviour Research Network (2012) Letter to the editor: Standardized use of the terms 'sedentary' and 'sedentary behaviours'. Appl Physiol Nutr Metab 37:540-542

21. Henry CJ (2005) Basal metabolic rate studies in humans: measurement and development of new equations. Public Health Nutr 8:11331152 
22. Horne R, Weinman J (1998) Predicting treatment adherence: an overview of theoretical models. In: Myers L, Midence K (eds) Adherence to treatment in medical conditions. Harwood Academic, London, pp 25-50

23. Bingham SA, Gill C, Welch A et al (1997) Validation of dietary assessment methods in the UK arm of EPIC using weighed records, and 24-hour urinary nitrogen and potassium and serum vitamin $\mathrm{C}$ and carotenoids as biomarkers. Int J Epidemiol 26:S137-S151

24. Office for National Statistics (2010) The National Statistics Socioeconomic Classification (NS-SEC rebased on the SOC2010). Available from www.ons.gov.uk/ons/guide-method/classifications/ current-standard-classifications/soc2010/soc2010-volume-3-ns-secrebased-on-soc2010-user-manual/index.html\#7, accessed 28 May 2013

25. Wareham NJ, Jakes RW, Rennie KL, Mitchell J, Hennings S, Day NE (2002) Validity and repeatability of the EPIC-Norfolk Physical Activity Questionnaire. Int J Epidemiol 31:168-174

26. Ekelund U, Griffin SJ, Wareham NJ (2007) Physical activity and metabolic risk in individuals with a family history of type 2 diabetes. Diabetes Care 30:337-342

27. Simmons RK, Griffin SJ, Steele R, Wareham NJ, Ekelund U, ProActive Research T (2008) Increasing overall physical activity and aerobic fitness is associated with improvements in metabolic risk: cohort analysis of the ProActive trial. Diabetologia 51:787-794

28. Healy GN, Dunstan DW, Salmon J et al (2007) Objectively measured light-intensity physical activity is independently associated with 2-h plasma glucose. Diabetes Care 30:1384-1389

29. Morselli L, Leproult R, Balbo M, Spiegel K (2010) Role of sleep duration in the regulation of glucose metabolism and appetite. Best Pract Res Clin Endocrinol Metab 24:687-702

30. Dunstan DW, Thorp AA, Healy GN (2011) Prolonged sitting: is it a distinct coronary heart disease risk factor? Curr Opin Cardiol 26: 412-419

31. Hamilton MT, Hamilton DG, Zderic TW (2007) Role of low energy expenditure and sitting in obesity, metabolic syndrome, type 2 diabetes, and cardiovascular disease. Diabetes 56:2655-2667

32. Borst C, Wieling W, van Brederode JF, Hond A, de Rijk LG, Dunning AJ (1982) Mechanisms of initial heart rate response to postural change. Am J Physiol 243:H676-H681

33. Wieling W, van Brederode JF, de Rijk LG, Borst C, Dunning AJ (1982) Reflex control of heart rate in normal subjects in relation to age: a data base for cardiac vagal neuropathy. Diabetologia 22:163-166

34. Healy GN, Wijndaele K, Dunstan DW et al (2008) Objectively measured sedentary time, physical activity, and metabolic risk: the Australian Diabetes, Obesity and Lifestyle Study (AusDiab). Diabetes Care 31:369-371

35. Ekelund U, Brage S, Franks PW, Hennings S, Emms S, Wareham NJ (2005) Physical activity energy expenditure predicts progression toward the metabolic syndrome independently of aerobic fitness in middle-aged healthy Caucasians: the Medical Research Council Ely Study. Diabetes Care 28:1195-1200
36. Helmerhorst HJ, Wijndaele K, Brage S, Wareham NJ, Ekelund U (2009) Objectively measured sedentary time may predict insulin resistance independent of moderate- and vigorous-intensity physical activity. Diabetes 58:1776-1779

37. Wareham NJ, Wong MY, Day NE (2000) Glucose intolerance and physical inactivity: the relative importance of low habitual energy expenditure and cardiorespiratory fitness. Am J Epidemiol 152:132139

38. Barengo NC, Hu G, Lakka TA, Pekkarinen H, Nissinen A, Tuomilehto J (2004) Low physical activity as a predictor for total and cardiovascular disease mortality in middle-aged men and women in Finland. Eur Heart J 25:2204-2211

39. Berlin JA, Colditz GA (1990) A meta-analysis of physical activity in the prevention of coronary heart disease. Am J Epidemiol 132:612 628

40. Lee IM, Paffenbarger RS Jr, Hennekens CH (1997) Physical activity, physical fitness and longevity. Aging (Milano) 9:2-11

41. Sattelmair J, Pertman J, Ding EL, Kohl HW 3rd, Haskell W, Lee IM (2011) Dose response between physical activity and risk of coronary heart disease: a meta-analysis. Circulation 124:789-795

42. Strath SJ, Brage S, Ekelund U (2005) Integration of physiological and accelerometer data to improve physical activity assessment. Med Sci Sports Exerc 37:S563-S571

43. Assah FK, Ekelund U, Brage S, Wright A, Mbanya JC, Wareham NJ (2011) Accuracy and validity of a combined heart rate and motion sensor for the measurement of free-living physical activity energy expenditure in adults in Cameroon. Int J Epidemiol 40:112-120

44. de Koning L, Merchant AT, Pogue J, Anand SS (2007) Waist circumference and waist-to-hip ratio as predictors of cardiovascular events: meta-regression analysis of prospective studies. Eur Heart J $28: 850-856$

45. Austin MA, Hokanson JE, Edwards KL (1998) Hypertriglyceridemia as a cardiovascular risk factor. Am J Cardiol 81:7B-12B

46. Voight BF, Peloso GM, Orho-Melander M et al (2012) Plasma HDL cholesterol and risk of myocardial infarction: a Mendelian randomisation study. Lancet 380:572-580

47. Katzmarzyk PT (2010) Physical activity, sedentary behavior, and health: paradigm paralysis or paradigm shift? Diabetes 59:27172725

48. Owen N, Healy GN, Matthews CE, Dunstan DW (2010) Too much sitting: the population health science of sedentary behavior. Exerc Sport Sci Rev 38:105-113

49. Chief Medical Officers (2011) Start active, stay active: a report on physical activity from the four home countries' chief medical officers. Available from https://www.gov.uk/government/publications/ start-active-stay-active-a-report-on-physical-activity-from-the-fourhome-countries-chief-medical-officers, accessed 20 May 2013

50. Bravata DM, Smith-Spangler C, Sundaram V et al (2007) Using pedometers to increase physical activity and improve health: a systematic review. JAMA 298:2296-2304 